Research Article

\title{
Reliability and Validity of the Korean Version of the Multidimensional Fatigue Inventory (MFI-20): A Multicenter, Cross-Sectional Study
}

\author{
Sang-Wook Song $\mathbb{D}^{1},{ }^{1}$ Sung-Goo Kang $\mathbb{D},{ }^{1}$ Kyung-Soo Kim, ${ }^{2}$ Moon-Jong Kim, ${ }^{3}$ \\ Kwang-Min Kim, ${ }^{4}$ Doo-Yeoun Cho, ${ }^{3}$ Young-Sang Kim $\left(\mathbb{D},{ }^{3}\right.$ Nam-Seok Joo, ${ }^{4}$ \\ and Kyu-Nam Kim (iD) \\ ${ }^{1}$ Department of Family Medicine, St. Vincent's Hospital, College of Medicine, The Catholic University of Korea, Suwon, \\ Republic of Korea \\ ${ }^{2}$ Department of Family Medicine, CMC Clinical Research Coordinating Center, Seoul St. Mary's Hospital, \\ College of Medicine, The Catholic University of Korea, Seoul, Republic of Korea \\ ${ }^{3}$ Department of Family Medicine, CHA Bundang Medical Center, CHA Medical University, Seongnam, Republic of Korea \\ ${ }^{4}$ Department of Family Practice and Community Health, Ajou University School of Medicine, Suwon, Republic of Korea
}

Correspondence should be addressed to Sung-Goo Kang; hippo94@naver.com

Received 2 February 2018; Revised 16 March 2018; Accepted 5 April 2018; Published 9 May 2018

Academic Editor: Bruno Gagnon

Copyright (c) 2018 Sang-Wook Song et al. This is an open access article distributed under the Creative Commons Attribution License, which permits unrestricted use, distribution, and reproduction in any medium, provided the original work is properly cited.

\begin{abstract}
Introduction. A nonspecific symptom, fatigue accompanies a variety of diseases, including cancer, and can have a grave impact on patients' quality of life. As for multidimensional instruments, one of the most widely used is the Multidimensional Fatigue Inventory (MFI). This study aims to verify the reliability and validity of the MFI Korean (MFI-K) version. Materials and Method. This study was performed at four university hospitals in the Republic of Korea. Among outpatients visiting the Department of Family Medicine, those complaining of fatigue or visiting a chronic care clinic were enrolled in this study. A total of 595 participants were included, and the mean age was 42.2 years. Results. The Cronbach's alpha coefficient of the MFI-K was 0.88 . The MFI-K had good convergent validity. Most subscales of the MFI-K were significantly correlated with the Visual Analogue Scale (VAS) and Fatigue Severity Scale (FSS). In particular, general and physical fatigue had the greatest correlation with the VAS and FSS. Although the English version of MFI had five subscales, the factor analysis led to four subscales in the Korean version. Conclusion. This study demonstrated the clinical usefulness of MFI-K instrument, particularly in assessing the degree of fatigue and performing a multidimensional assessment of fatigue.
\end{abstract}

\section{Introduction}

Fatigue is a largely subjective symptom and is one of the most common symptoms encountered in primary care. A nonspecific symptom, fatigue accompanies a variety of diseases, including cancer, and can have a grave impact on patients' quality of life [1-8]. The medical cause of fatigue remains undiscovered in about $59-64 \%$ of adults who claim to have it [1]. Because of this nonspecificity, differential diagnosis and management of fatigue is often difficult. Nevertheless, both patients and physicians alike tend to underestimate fatigue symptoms solely due to their high prevalence.

One study showed that the prevalence of fatigue varies widely in primary practice, from 7 to $45 \%$. One reason for such wide variation might be its ambiguous definition and differences in measurement instruments used [1].

Instruments useful for assessing fatigue can be categorized as unidimensional and multidimensional. The most widely used unidimensional fatigue assessment instruments are the Visual Analogue Scale (VAS) and Fatigue Severity Scale (FSS) developed by Dr. Krupp $[9,10]$. The FSS has 
already been translated into Korean and validated [11]. As for multidimensional instruments, one of the most widely used is the Multidimensional Fatigue Inventory (MFI) [12]. The MFI was developed by E. M. A. Smets, a Dutch doctor. The validities of the English [12], French, and Swedish versions $[13,14]$ of the scale have been verified. The MFI has five subscales: general fatigue, physical fatigue, reduced activity, reduced motivation, and mental fatigue. Gentile et al. [13] conducted factor analysis at the same time as they conducted a validation study of the French version of MFI. The subscale of the French version of MFI was different from that of the MFI of the original version. The structure of Korean sentences is very different from the structure of English sentences. Korean sentences have the structure of subject + object + verb. Even the meaning of positive and negative is placed at the end of the sentence. Therefore, the subscales of MFI Korean version are very likely to be different from the MFI original version.

Because of cultural differences, translated scales must be appropriately validated to be suitable to the corresponding culture. Although many studies on fatigue in various patient populations, including patients with cancer and COPD, are underway in South Korea, there is practically no multidimensional instrument with verified reliability and validity in Korean. While researchers have translated the MFI into Korean and used it in practice, the reliability and validity of the translated version have not been established. Therefore, this study aims to verify the reliability and validity of the MFI Korean (MFI-K) version and to establish a new factor structure for the Korean outpatient population.

\section{Materials and Methods}

2.1. Study Population. The study population comprised adults aged 19 years or older. This study was performed at four university hospitals based in Seoul $(n=1)$ and two cities in Gyeonggi Province: Suwon $(n=2)$ and Seongnam $(n=1)$. Since Seoul is a metropolitan city with a population of more than 10 million, and Seongnam and Suwon are also cities with populations of more than 1 million, the subjects of this study were all urban people. Among outpatients visiting the Department of Family Medicine, those complaining of fatigue or who were visiting a chronic care clinic were enrolled in this study. Individuals without fatigue symptoms who have visited the health improvement center for a routine health examination were established as the control group. On the first page of the questionnaire, participants were asked to choose one of the following responses regarding their level of fatigue over the past week, before continuing with the rest of the questionnaire: (1) rarely, (2) moderate, and (3) very severe.

2.2. Translation of $M F I-K$. The translation was performed after obtaining approval from Dr. Smets, the developer of the MFI. The translation process suggested by Guillemin et al. [15] and Beaton et al. [16] was followed. First, two Korean native translators proficient in English independently translated the English version into Korean. One of these translators was the first author of this study, and the other translator was blinded to the purpose of the questionnaire to enhance the quality of the translation. Next, four specialists discussed and analyzed the differences between the two translations to merge them into a single complete translation. During the back-translation stage, two new back-translators independently translated the completed translation into the source language (English). The back-translators were native English speakers also proficient in Korean. In addition, they were not medical professionals and were blinded to the purpose of the questionnaire. Finally, four specialists discussed and analyzed the back-translated version to complete the final MFI-K. Dr. Smets reviewed the final version and approved the study using MFI-K.

2.3. Visual Analogue Scale (VAS). The VAS is $100 \mathrm{~mm}$ long, with the left end indicating "no exhaustion at all" and the right end indicating "complete exhaustion." We showed participants the VAS and instructed them to put a mark on the VAS line to indicate their level of fatigue. Then we measured the length $(\mathrm{mm})$ from the left end of the line to the mark to indicate their score. A higher score indicated more severe fatigue.

2.4. Fatigue Severity Scale (FSS). To assess fatigue severity, we additionally used the FSS developed by Krupp et al. [10]. The FSS is a 9-item self-report questionnaire. Each item is rated on a seven-point scale, where respondents choose a score between 1 (no impairment at all) and 7 (severe impairment) corresponding to their perceived impairment. The total score is then divided by the total number of items to generate the mean score. This scale was translated into Korean previously and has been found to be clinically useful [11].

2.5. Statistical Analysis. All statistical analyses were performed using the PASW Statistics (i.e., SPSS) 18.0 for Windows. Items within the original five factors of the MFI-K were assessed for their internal consistency by using Cronbach's alpha. Principal component analysis was performed to examine the MFI-K's factor structure; factors with eigenvalues of $>1$ were extracted. The convergent validity of the total cognition score was tested using the Spearman rank-order correlation and Pearson correlation coefficients with the VAS and FSS. Differences between the fatigue groups were tested using the chi-square test and ANOVA.

2.6. Ethics Statement. This study was implemented in accordance with ethical and safety guidelines upon the approval of the Institutional Review Board in The Catholic University of Korea, St. Vincent's Hospital (IRB approval number: VC15QIMI0193). We provided adequate explanation of the aim, structure, content, and precautions of the study to all participants, and participants were given sufficient time to make decisions. The participants were then instructed to answer all questionnaire items after reading the instructions of each instrument. They were told to ask any questions they might have during the course of completing 
TABLE 1: Baseline characteristics of the study population.

\begin{tabular}{|c|c|c|c|c|}
\hline & No or mild fatigue $(N=76)$ & Moderate fatigue $(N=342)$ & Severe fatigue $(N=174)$ & $P$ value \\
\hline Age (years) & $45.39 \pm 13.68$ & $42.39 \pm 12.20$ & $40.49 \pm 11.82$ & 0.014 \\
\hline Sex & & & & 0.071 \\
\hline Male & $36(16.8)$ & $122(57.0)$ & $56(26.2)$ & \\
\hline Female & $40(10.6)$ & $222(28.6)$ & $117(30.9)$ & \\
\hline BMI $\left(\mathrm{kg} / \mathrm{m}^{2}\right)$ & $24.04 \pm 5.36$ & $23.39 \pm 10.01$ & $22.79 \pm 3.61$ & 0.506 \\
\hline Sleep duration & $6.78 \pm 1.09$ & $6.43 \pm 1.08$ & $6.23 \pm 1.31$ & 0.002 \\
\hline Smoking & & & & 0.161 \\
\hline Smoker & $9(14.1)$ & $31(48.4)$ & $25(37.5)$ & \\
\hline Ex-smoker & $16(19.8)$ & $44(54.3)$ & $21(25.9)$ & \\
\hline Nonsmoker & $50(11.4)$ & $260(59.5)$ & $127(29.1)$ & \\
\hline Marital condition & & & & 0.542 \\
\hline Single & $18(10.7)$ & $98(58.0)$ & $53(31.4)$ & \\
\hline Married & $56(13.9)$ & $231(57.3)$ & $116(28.8)$ & \\
\hline Others & $4(19.0)$ & $13(61.9)$ & $4(19.0)$ & \\
\hline Education & & & & 0.362 \\
\hline Under middle school & $6(17.6)$ & $19(55.9)$ & $9(26.5)$ & \\
\hline High school or college & $37(17.3)$ & $119(55.6)$ & $58(27.1)$ & \\
\hline Over university & $35(10.1)$ & $205(59.2)$ & $106(30.6)$ & \\
\hline
\end{tabular}

Others: separation, divorce, or bereavement, data are mean \pm SD or $N(\%)$; $P$ values were obtained by the one-way ANOVA or chi-square test.

Table 2: Principal component analysis after varimax rotation of MFI Korean version.

\begin{tabular}{lcccc}
\hline Factors & Eigenvalue & \% total of variance & Items & Interpretation of Korean dimensions \\
\hline Factor 1 & 6.37 & 31.85 & $1,5,12,14,16,20$ & General and physical fatigue \\
Factor 2 & 2.48 & 6.42 & $7,9,11,13,18,19$ & Mental fatigue \\
Factor 3 & 1.38 & 6.15 & $2,6,10,17$ & Reduced activity \\
Factor 4 & 1.23 & $3,4,8,15$ & Motivation \\
\hline
\end{tabular}

the questionnaire to ensure that they accurately understand the instrument items.

\section{Results}

3.1. General Characteristics. A total of 595 participants were included, and the mean age was $42.22 \pm 12.36$ years. A total of $380(63.99 \%)$ participants were women and $215(36.01 \%)$ were men. The study groups did not significantly differ in terms of body mass index, marital status, highest education level, and smoking status. Although statistically nonsignificant, women tended to experience more severe fatigue than did men. Participants who reported severe fatigue were younger and slept significantly less (Table 1).

Fifty-one (8.5\%) participants said that the content of the questionnaire was slightly difficult to understand, while 8 participants (1.3\%) said that the content of the questionnaire was very difficult to understand. The remaining 538 (90.2\%) participants said that the content of the questionnaire was neither difficult nor easy to understand or was easy to understand.

Overall, the MFI-K was well received by the participants. About $99.5 \%$ of the participants completed the MFI-K without omissions. Extremely few questionnaires were submitted with missing items (less than $0.5 \%$ ).

The time taken to complete the MFI-K did not significantly differ among the fatigue groups $(4.14 \pm 2.95$ versus $4.05 \pm 3.25$ versus $4.36 \pm 3.06, P=0.557)$.
3.2. Internal Consistency. The Cronbach's alpha coefficient of the MFI-K was 0.88. The internal consistency of each of the five original subscales was as follows: general fatigue (0.84), physical fatigue (0.78), mental fatigue (0.67), reduced activity (0.66), and reduced motivation (0.52).

3.3. Structure Validity. The results of the principal component analysis (with a varimax rotation) are shown in Tables 2 and 3. Four factors explained $57.33 \%$ of the total variance. Factor 1 explains $31.9 \%$ of the total variance and included all of the "general fatigue" items and some "physical fatigue" items. Factor 2 explains $12.4 \%$ of the total variance and includes all "mental fatigue" items and "I don't feel like doing anything" items. Factor 3 mostly includes the "reduced activities" items, and most of the items are phrased negatively. Factor 4 included positively worded phrases about motivation. Although the English version had five subscales, the factor analysis led to four subscales in the Korean version.

3.4. Convergent Validity. The MFI-K had good convergent validity. Most subscales of the MFI-K were significantly correlated with the VAS. In particular, general fatigue had the greatest correlation with the VAS $(r=0.533)$. Reduced activity had the lowest significant correlation with the VAS $(r=0.084)$.

Similarly, the general fatigue subscale of the MFI-K had the strongest correlation with the FSS $(r=0.725)$. 
TABLE 3: Items of the new dimension of the MFI Korean version.



The reduced activity subscale had the lowest correlation with the FSS $(r=0.162)$ but the correlation was statistically significant (Table 4). These results were also found for the new factor structure of the MFI-K (Tables 5 and 6).

3.5. Differences in MFI-K Scores across Fatigue Groups. The mean MFI-K score significantly varied across the fatigue groups. With the exception of reduced activities, all subgroup scores significantly differed across the groups. The VAS and FSS scores also significantly differed across groups. In all cases, the mean MFI-K score increased with increasing fatigue severity (Table 6).
TABLE 4: Correlation between MFI-K and VAS score and FSS score in English factor structure.

\begin{tabular}{lcc}
\hline Dimensions: English structure & VAS score & FSS score \\
\hline General fatigue & $0.533^{* *}$ & $0.725^{* *}$ \\
Physical fatigue & $0.378^{* *}$ & $0.578^{* *}$ \\
Mental fatigue & $0.241^{* *}$ & $0.403^{* *}$ \\
Reduced activity & $0.084^{*}$ & $0.162^{* *}$ \\
Reduced motivation & $0.276^{* *}$ & $0.391^{* *}$ \\
MFI-K total score & $0.419^{* *}$ & $0.635^{* *}$ \\
\hline
\end{tabular}

${ }^{* *} P<0.01,{ }^{*} P<0.05$; correlation coefficients were obtained by Spearman correlation analysis.

TABLE 5: Correlation between MFI-K and VAS score and FSS score in Korean factor structure.

\begin{tabular}{lcc}
\hline Dimensions: Korean structure & VAS score & FSS score \\
\hline General and physical fatigue & $0.519^{* *}$ & $0.718^{* *}$ \\
Mental fatigue & $0.333^{* *}$ & $0.506^{* *}$ \\
Reduced activity & $0.087^{*}$ & $0.200^{* *}$ \\
Motivation & $0.159^{* *}$ & $0.248^{* *}$ \\
MFI-K total score & $0.419^{* *}$ & $0.635^{* *}$ \\
\hline
\end{tabular}

${ }^{* *} P<0.01,{ }^{*} P<0.05$; correlation coefficients were obtained by Spearman correlation analysis.

\section{Discussion}

This study aimed to verify the reliability and validity of the Korean version of the MFI, one of the most widely used multidimensional fatigue scales. It was also the first study to examine a Korean version of the Multidimensional Fatigue Scale.

We tested the reliability of the MFI-K using Cronbach's alpha coefficients. Scales with Cronbach's alpha values of greater than 0.7 are considered reliable [17], and the Cronbach's alpha coefficient of the MFI-K was 0.88 , suggesting high reliability. The subscales also showed reasonable internal consistency $(>0.65)$, with the exception of reduced motivation. This was similar to the results presented by Dr. Smets, the developer of the MFI [12]. However, the internal consistency was generally lower than that of the French version of the MFI [13]. Although additional studies are needed to shed light on the causes of the difference between our findings and findings from other countries, it is nevertheless possible that differences in sentence structures are involved. For instance, unlike English and French, the verb is placed at the end of the sentence in Korean, which means that words suggesting a negative or positive tone are placed at the end of the sentence, requiring readers to focus on the end of each sentence in order to accurately understand the whole sentence. As proof of this, factor 3 mostly includes negatively worded phrases (except one item, 6, "I think I do a lot in a day") and factor 4 included positively worded phrases.

To know this more clearly, further research in Japan and China is needed to confirm whether this is a geographical, cultural, or sentence structural problem. Although Korea, China, and Japan have very close geographical and historical relations, the language structure of Chinese is similar to English, but the language structure of Japanese is similar to 
TABLE 6: Univariate F-test for group differences on subclass of the MFI Korean structure.

\begin{tabular}{|c|c|c|c|c|}
\hline & No or mild fatigue & Moderate fatigue & Severe fatigue & $P$ value \\
\hline \multicolumn{5}{|l|}{ MFI Korean structure } \\
\hline General and physical fatigue & $14.37 \pm 4.73$ & $20.67 \pm 4.30$ & $26.83 \pm 3.94$ & $<0.001$ \\
\hline Mental fatigue & $9.66 \pm 2.62$ & $12.17 \pm 3.13$ & $14.07 \pm 3.78$ & $<0.001$ \\
\hline Reduced activities & $8.46 \pm 3.33$ & $9.12 \pm 2.54$ & $9.22 \pm 3.23$ & 0.131 \\
\hline Motivation & $8.94 \pm 3.14$ & $10.87 \pm 2.76$ & $11.11 \pm 3.64$ & $<0.001$ \\
\hline MFI-K total score & $41.36 \pm 10.52$ & $52.85 \pm 9.63$ & $61.23 \pm 10.54$ & $<0.001$ \\
\hline VAS score & $16.85 \pm 18.19$ & $33.02 \pm 26.53$ & $55.74 \pm 32.28$ & $<0.001$ \\
\hline FSS score & $20.59 \pm 9.09$ & $31.26 \pm 10.25$ & $43.81 \pm 10.25$ & $<0.001$ \\
\hline
\end{tabular}

Korean. Therefore, if MFI is translated into Chinese and Japanese, and factor analysis is performed, it is possible to know more exactly whether MFI-K differs from MFI of English version because of geographical and cultural differences or differences in language structure.

As anticipated, the general and physical fatigue subscale of the MFI-K had the strongest correlations with the VAS and FSS. This is in line with the findings of Dr. Smets [18] that the general fatigue subscale of the MFI-20 is compatible with the VAS for assessing fatigue in cancer patients, and with the findings of Rupp et al. [4] that the general fatigue and physical fatigue subscales of the MFI-20 had the highest correlations with the VAS in patients with rheumatoid arthritis. The subjects of this study were people who visited family medicine departments for (generally physical) fatigue or visited health promotion centers for routine health checkups. The correlation between general and physical fatigue subscale of the MFI-K and FSS was higher than 0.7 and showed a low correlation with other subscales. These results demonstrate the ability of the MFI-K to differentiate between the degree and the type of fatigue.

As for the structural validity, the MFI-K has only four subscales (Tables 2 and 3), whereas the English version has five. The English version of the MFI includes general fatigue (items 1, 5, 12, and 16), physical fatigue (items 2, 8, 14, and 20), reduced activity (items $3,6,10$, and 17), reduced motivation (item 4, 9, 15, and 18), and mental fatigue (item $7,11,13$, and 19) subscales (Table 3). In our study, it was difficult to distinguish general fatigue from physical fatigue, which is similar to the study by Dr. Smets and the study on the Swedish version of the MFI [19]. With the exception of item 2 and item 8, items corresponding to general fatigue and physical fatigue in the English version of MFI consisted of one factor (general and physical fatigue) in MFI-K. In the English version of MFI, item 2 and item 8 are included in the physical fatigue subscale. However, in MFI-K, item 2 ("Physically I feel only able to do a little") was more relevant to the reduced activities subscale, and item 8 ("Physically I can take on a lot") was more relevant to the motivation subscale. In the English version of MFI, item 9 and item 18 are included in the reduced motivation subscale. In MFI-K, item 9 ("I dread having to do things") and item 18 ("I don't feel like doing anything") were more relevant to the mental fatigue subscale. As mentioned earlier, in MFI-K, factor 3 (reduced activity) mostly included negatively worded phrases (except one item, 6, "I think I do a lot in a day") and factor 4 (motivation) included positively worded phrases. It is a sentence of the same meaning, but depending on the English or Korean language, it can be conveyed to the person reading the item with a different feeling. In the end, the structure of the MFI-K was generally inconsistent with the English version of the MFI and was closer to the French version.

This study has a few limitations. First, the subjects of this study were all urban people. Rural people were not included in the study. However, as of $2016,91.8 \%$ of the total population of Korea lives in cities [20]. Second, in this study, the employment status of the subjects was not investigated.

This study demonstrated the clinical usefulness of this instrument, particularly in assessing the degree of fatigue and performing a multidimensional assessment of fatigue. The general and physical fatigue subscale was strongly associated with the global fatigue measures (VAS and FSS), and the other subscales had fair relationships with the FSS. These results show that fatigue is a multidimensional concept and various aspects must be considered when dealing with patients with fatigue. Follow-up studies should be conducted on a more diverse study population for whom fatigue assessment using the MFI-K is important, such as cancer patients or families of cancer patients, as well as patients with chronic fatigue syndrome, fibromyalgia, or chronic obstructive pulmonary disease.

\section{Conclusion}

This study demonstrated the clinical usefulness of MFI-K instrument, particularly in assessing the degree of fatigue and performing a multidimensional assessment of fatigue. The MFI-K is the first validated tool to assess various aspects of fatigue in Koreans.

\section{Data Availability}

The data used to support the findings of this study are available from the corresponding author upon request.

\section{Conflicts of Interest}

All authors declare that they have no relevant conflicts of interest.

\section{Funding}

This study was supported by the Daewoong Pharmaceutical company. 


\section{Acknowledgments}

The authors sincerely thank Dr. E. M. A. Smets for allowing the Korean version of MFI to be studied.

\section{References}

[1] G. Lewis and S. Wessely, "The epidemiology of fatigue: more questions than answers," Journal of Epidemiology and Community Health, vol. 46, no. 2, pp. 92-97, 1992.

[2] J. E. Bower, K. Bak, A. Berger et al., "Screening, assessment, and management of fatigue in adult survivors of cancer: an American Society of Clinical oncology clinical practice guideline adaptation," Journal of Clinical Oncology, vol. 32, no. 17, pp. 1840-1850, 2014.

[3] J. P. Solano, B. Gomes, and I. J. Higginson, "A comparison of symptom prevalence in far advanced cancer, AIDS, heart disease, chronic obstructive pulmonary disease and renal disease," Journal of Pain and Symptom Management, vol. 31, no. 1, pp. 58-69, 2006.

[4] I. Rupp, H. C. Boshuizen, C. E. Jacobi, H. J. Dinant, and G. A. van den Bos, "Impact of fatigue on health-related quality of life in rheumatoid arthritis," Arthritis and Rheumatology, vol. 51, no. 4, pp. 578-585, 2004.

[5] S. O. Breukink, J. H. Strijbos, M. Koorn, G. H. Koeter, E. H. Breslin, and C. P. van der Schans, "Relationship between subjective fatigue and physiological variables in patients with chronic obstructive pulmonary disease," Respiratory Medicine, vol. 92, no. 4, pp. 676-682, 1998.

[6] M. J. Hjermstad, P. M. Fayers, K. Bjordal, and S. Kaasa, "Health-related quality of life in the general Norwegian population assessed by the European Organization for Research and Treatment of Cancer Core Quality-of-Life Questionnaire: The QLQ=C30 (+3)," Journal of Clinical Oncology, vol. 16, no. 3, pp. 1188-1196, 1998.

[7] H. Michelson, C. Bolund, B. Nilsson, and Y. Brandberg, "Healthrelated quality of life measured by the EORTC QLQC30 reference values from a large sample of Swedish population," Acta Oncologica, vol. 39, no. 4, pp. 477-484, 2000.

[8] M. Lavidor, A. Weller, and H. Babkoff, "Multidimensional fatigue, somatic symptoms and depression," British Journal of Health Psychology, vol. 7, no. 1, pp. 67-75, 2002.

[9] L. B. Krupp, L. A. Alvarez, N. G. LaRocca, and L. C. Scheinberg, "Fatigue in multiple sclerosis," Archives of Neurology, vol. 45, no. 4, pp. 435-437, 1988.

[10] L. B. Krupp, N. G. LaRocca, J. Muir-Nash, and A. D. Steinberg, "The fatigue severity scale application to patients with multiple sclerosis and systemic lupus erythematosus," Archives of Neurology, vol. 46, no. 10, pp. 1121-1123, 1989.

[11] K. I. Chung and C. H. Song, "Clinical usefulness of fatigue severity scale for patients with fatigue, and anxiety or depression," Korean Journal of Psychosomatic Medicine, vol. 9, no. 2, pp. 164-173, 2001.

[12] E. M. A. Smets, B. Garssen, B. Bonke, and J. C. J. M. De Haes, "The Multidimensional Fatigue Inventory (MFI) psychometric qualities of an instrument to assess fatigue," Journal of Psychosomatic Research, vol. 39, no. 3, pp. 315-325, 1995.

[13] S. Gentile, J. C. Delarozière, F. Favre, R. Sambuc, and J. L. San Marco, "Validation of the French 'multidimensional fatigue inventory' (MFI 20)," European Journal of Cancer Care, vol. 12, no. 1, pp. 58-64, 2003.

[14] A. Ericsson and K. Mannerkorpi, "Assessment of fatigue in patients with fibromyalgia and chronic widespread pain. Reliability and validity of the Swedish version of the MFI-20,"
Disability and Rehabilitation, vol. 29, no. 22, pp. 1665-1670, 2007.

[15] F. Guillemin, C. Bombardier, and D. Beaton, "Cross-cultural adaptation of health related quality of life measure: literature review and proposed guidelines," Journal of Clinical Epidemiology, vol. 46, pp. 1417-1432, 1993.

[16] D. E. Beaton, C. Bombardier, F. Guillemin, and M. B. Ferraz, "Guidelines for the process of cross cultural adaptation of self report measures," Spine, vol. 25, no. 24, pp. 3186-3191, 2000.

[17] L. J. Cronbach, "Coefficient alpha and the internal structure of tests," Psychometrika, vol. 16, no. 3, pp. 297-334, 1951.

[18] C. L. Hagelin, Y. Wengström, S. Runesdotter, and C. J. Fürst, "The psychometric properties of the Swedish Multidimensional Fatigue Inventory MFI-20 in four different populations," Acta Oncologica, vol. 46, no. 1, pp. 97-104, 2007.

[19] E. M. Smets, B. Garssen, A. Cull, and J. C. de Haes, "Application of the multidimensional fatigue inventory (MFI-20) in cancer patients receiving radiotherapy," British Journal of Cancer, vol. 73, no. 2, pp. 241-245, 1996.

[20] Ministry of Land, Infrastructure and Transport (Republic of Korea), http://www.index.go.kr/potal/main/EachDtlPageDetail. do?idx_cd=1200. 




The Scientific World Journal
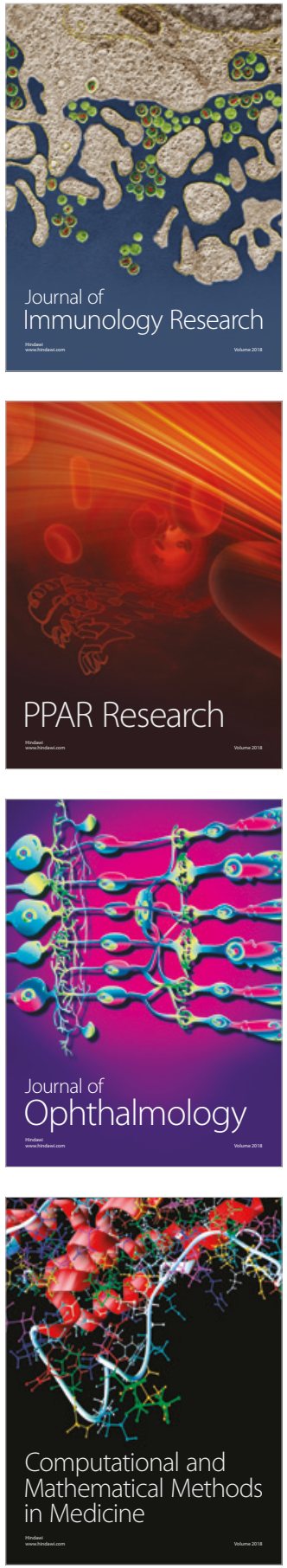

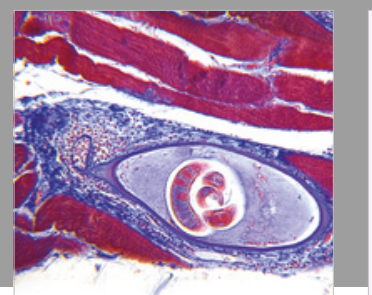

Gastroenterology Research and Practice

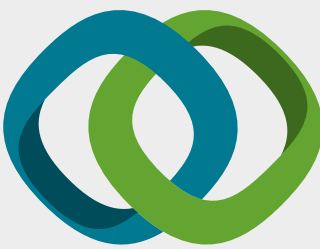

\section{Hindawi}

Submit your manuscripts at

www.hindawi.com


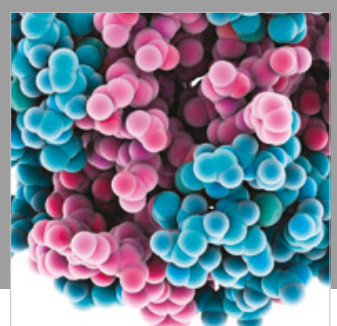

ournal of

Diabetes Research

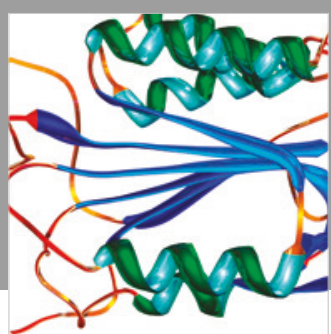

Disease Markers
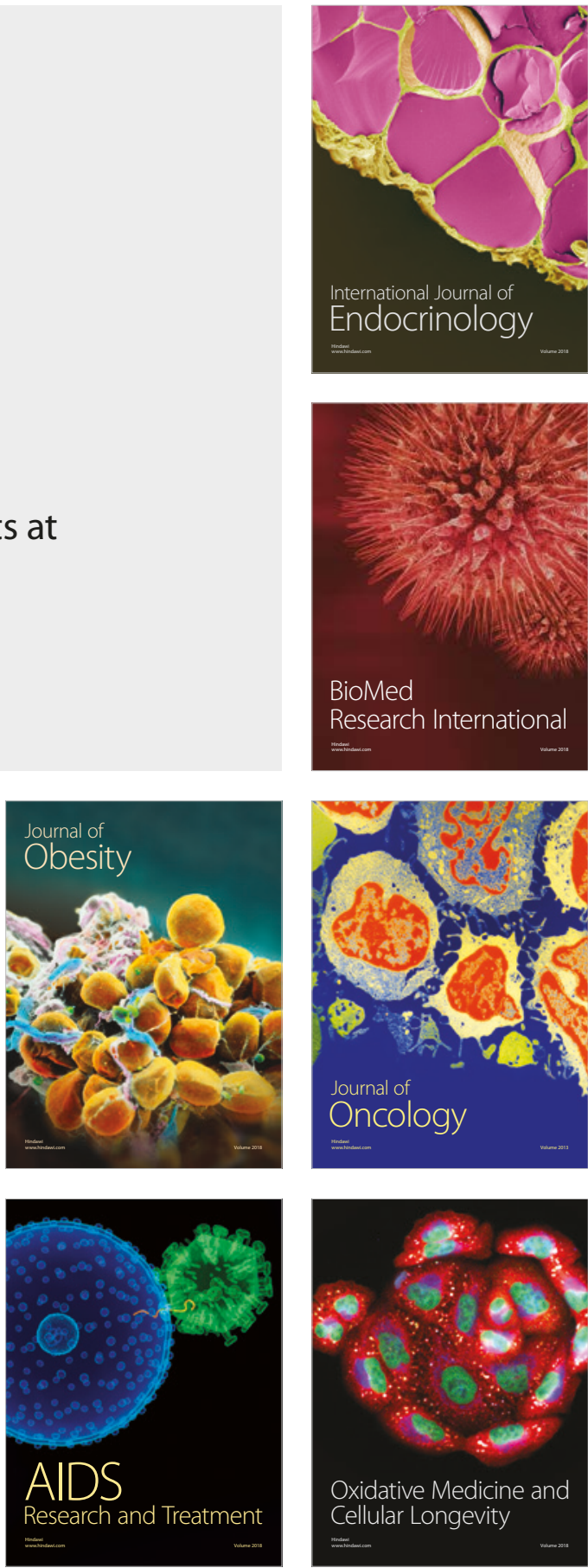